\title{
Generation of Steady Liquid Microthreads and Micron-Sized Monodisperse Sprays in Gas Streams
}

\author{
Alfonso M. Gañán-Calvo \\ Escuela Superior de Ingenieros, Universidad de Sevilla, Camino de los Descubrimientos s/n, 41092 Sevilla, Spain
}

(Received 14 August 1997)

\begin{abstract}
A new class of microscopic jet flows is here reported: for a certain range of physical parameters and geometrical configurations, a perfectly steady microscopic liquid thread can be formed by a laminar accelerating gas stream, eventually giving rise to a nearly monodisperse fine spray. Some interesting characteristics for many applications of this robust and versatile flow and related atomization technique are highlighted. Concentric multicomponent liquid threads can also be produced. A theoretical model is presented that shows agreement with experiments. [S0031-9007(97)04921-1]
\end{abstract}

PACS numbers: 47.55.Dz, 47.15.Hg, 47.27.Wg

Many economically vital processes in industry, technology, and medicine (e.g., combustion, agricultural treatment, surface finishing, respiratory drug delivery, atomic spectrometry, etc.) rely on the aerosol production from liquids. In the cases of drug inhalation or atomic spectroscopy the quality of the atomization process is even more critical. As general characteristics, the spray should be as fine, stable, monodisperse, reproducible, and controllable as possible [1].

The formation of liquid ligaments is in most cases a mandatory preliminary step for the production of a spray: common atomizers and nebulizers either employ a certain pressure to extrude the liquid, with the subsequent formation of small ligaments which eventually break up into droplets (direct pressure atomizers), or use a gas stream to promote the ejection of ligaments from a liquid-gas interface (air-assisted or pneumatic atomizers). Other methods involving alternative forces (electrostatic, centrifugal, or acoustic forces) are used in some applications for which specific characteristics (small size and/or monodispersity) are crucial. These last methods, however, possess serious shortcomings (i.e., noise, high electric charge, bulky systems, etc.).

In this work we report a microscopic moderate Reynolds number laminar flow which constitutes the physical basis for a novel pneumatic atomization technique [2] with applications in combustion, coating, microencapsulation, drug delivery, high quality powder or fiber production, etc. Basically, this flow is characterized by the formation of a steady microscopic liquid thread in the core of a highly accelerating (extensional) laminar gas stream. When the gas velocity is chosen so that gas pressure fluctuations are smaller than liquid-gas surface tension stresses, jet breakup yields a nearly monodisperse spray. We will show that the viscous shear stresses present in the liquid are minimal for this atomization procedure as compared to other mechanical methods (i.e., direct pressure, pneumatic, impinging jet, and other similar methods). This feature represents an additional advantage, essential for molecular biology, biochemistry analysis (e.g., [3]), or aerosol deliv- ery of medicines [4], which require in most cases careful or limited stress handling of small sample quantities of long and fragile molecular species.

Flow characteristics. - When a small liquid drop attached to the mouth of a capillary tube (with radius $R_{O}$ ) is located close to a small hole (with diameter $D$ ) in a thin plate (with thickness $L$ ) through which a gas stream is flowing, the drop elongates towards the hole. Let $H$ be the tube mouth to plate distance. $R_{o}, D, H$, and $L$ measure some hundreds microns. The drop develops a cusplike shape at a critical distance from the hole, when the applied pressure drop $\Delta P_{g}$ through the hole overcomes the liquid-gas surface tension stresses $O\left(\gamma / R^{*}\right)$ appearing at the point of maximum curvature, say $1 / R^{*}$ (close to the hole). Then, if the liquid flow rate $Q$ ejected from the drop cusp is steadily supplied from the capillary tube, a steady state is established [Fig. 1(a)]. A steady thin liquid jet with typical diameter $d_{j}$ is smoothly emitted from
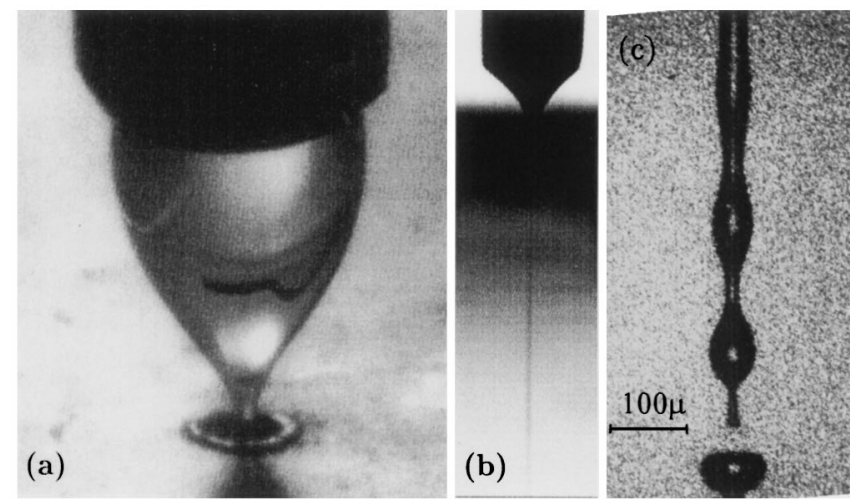

FIG. 1. (a) Typical view of the steady drop-jet mode. $R_{o}=$ $400 \mu \mathrm{m}, D=150 \mu \mathrm{m}, H=1 \mathrm{~mm}, L=300 \mu \mathrm{m}, \Delta P_{g}=$ $20 \mathrm{kPa}, Q=2$ liter s $^{-1}$. Liquid: water. (b) General view of the cone-jet configuration with the steady liquid thread crossing the plate. $R_{o}=200 \mu \mathrm{m}, D=150 \mu \mathrm{m}, L=350 \mu \mathrm{m}$, $\Delta P_{g}=5 \mathrm{kPa}, Q=0.5 \mu$ liter s$^{-1}$. Liquid: ethanol. (c) Closeup view of the breakup region taken with an ultrahigh speed video camera (shutter time $70 \mathrm{~ns}$ ), showing a highly regular axisymmetric breakup pattern. 
the cusplike drop shape and extends over distances of the order of millimeters depending on the liquid flow rate and the Reynolds number of the gas stream exiting the hole [see Fig. 1(b)]. The liquid jet shows a robust behavior provided that the pressure drop $\Delta P_{g}$ applied to the gas is sufficiently large compared to the maximum surface tension stress, of the order of $\gamma / d_{j}$, that act at the liquid-gas interface [see Fig. 1(b)], and is stable owing to the jet's slightly parabolic axial velocity profile [5].

Once the liquid jet exits the hole [Fig. 1(b)], the liquid pressure $P_{l}$ becomes (like the gas pressure $P_{g}$ ) almost constant in the axial direction, and the jet diameter remains almost constant up to the point where it breaks up by capillary instability [Fig. 1(c)]. Defining a Weber number $\mathrm{We}=\left(\rho_{g} v_{g}^{2} d_{j}\right) / \gamma \simeq 2 \Delta P_{g} d_{j} / \gamma$ (where $v_{g}$ is the gas velocity measured at the orifice), below a certain experimental value $\mathrm{We}_{c} \sim 40$ the breakup mode is axisymmetric and the resulting droplet stream is characterized by its monodispersity (Fig. 2), provided that the fluctuations of the gas flow do not contribute to droplet coalescence (these fluctuations occur when the gas stream reaches a fully developed turbulent profile around the liquid jet breakup region). Above this $\mathrm{We}_{c}$ value, sinuous nonaxisymmetric disturbances become apparent, coupled to the axisymmetric ones. For larger We numbers, the nonlinear growth rate of the sinuous ones seems to overcome that of the axisymmetric ones. The resulting spray shows significant polydispersity in this case (Fig. 3).

Hydrodynamics and universal diameter of the liquid jet.-We assume cylindrical coordinates $(r, z)$ for the analysis of the thread dynamics. The cusplike meniscus formed at the tube's mouth is pulled towards the hole by the pressure gradient created by the gas stream. From the cusp of this meniscus, a steady liquid thread with shape of radius $r=\xi$ is withdrawn through the hole by the action of both the suction effect due to $\Delta P_{g}$, and the tangential

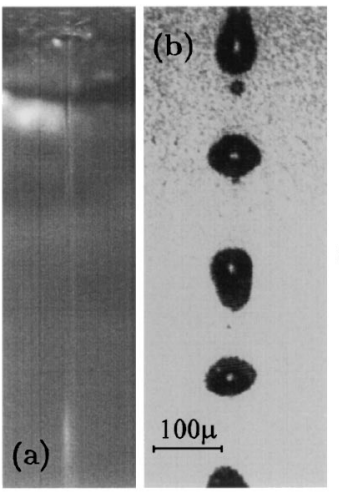

(c)

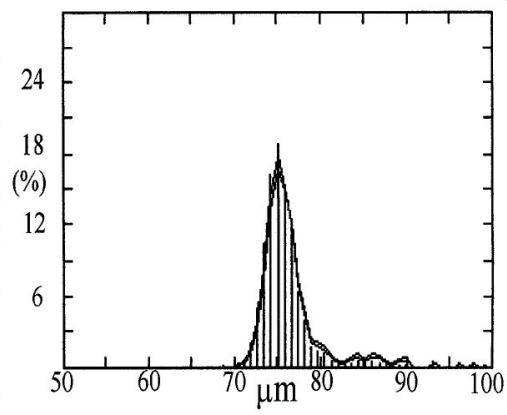

FIG. 2. (a) View of microjet near the exit of the orifice. $d_{j}=46 \mu \mathrm{m} ; D=150 \mu \mathrm{m}, L=100 \mu \mathrm{m} ; \Delta P_{g}=4.67 \mathrm{kPa}$, and $Q=4.9 \mu$ liter/s. Liquid: water. (b) Close-up view of the breakup region, taken with an ultrahigh speed video camera. This $70 \mathrm{~ns}$ photograph shows a regular breakup pattern. (c) Droplet size histogram showing its uniformity, measured at $20 \mathrm{~mm}$ of the orifice. Standard deviation $\sigma=6.7 \%$. viscous stresses $\tau_{s}$ exerted by the gas on the jet's surface in the axial direction. The averaged momentum equation for this configuration may be written (e.g., [6-10]):

$$
\frac{d}{d z}\left[P_{l}+\frac{\rho_{l} Q^{2}}{2 \pi^{2} \xi^{4}}\right]=\frac{2 \tau_{s}}{\xi},
$$

assuming that the viscous extensional term $[8,10]$ is negligible compared to the kinetic energy term, as will be subsequently justified. In addition, liquid evaporation is neglected. The liquid pressure $P_{l}$ is given by the capillary equation

$$
P_{l}=P_{g}+\gamma / \xi .
$$

As shown by the experiments, the pressure drop $\Delta P_{g}$ is sufficiently large as compared to the surface tension stress $\gamma / \xi$ to neglect the latter in the analysis. This scenario holds for the whole range of flow rates in which the thread is absolutely stable. In fact, it will be shown that, for a given pressure drop $\Delta P_{g}$, the minimum liquid flow rate that can be sprayed in steady jet conditions is achieved when the surface tension stress $\gamma / \xi$ is of the order of the kinetic energy of the liquid $\rho_{l} Q^{2} /\left(2 \pi^{2} \xi^{4}\right)$, since the surface tension acts like a "resistance" to the motion [it appears as a negative term in the right-hand side term of Eq. (1)]. Thus,

$$
Q_{\min } \sim\left(\frac{\gamma d_{j}^{3}}{\rho_{l}}\right)^{1 / 2}
$$

For sufficiently large flow rates $Q$ compared to $Q_{\min }$, the simplified averaged momentum equation in the axial direction can be expressed as

$$
\frac{d}{d z}\left(\frac{\rho_{l} Q^{2}}{2 \pi^{2} \xi^{4}}\right)=-\frac{d P_{g}}{d z}+\frac{2 \tau_{s}}{\xi},
$$

where one can identify the two driving forces for the liquid flow on the right-hand side. This equation can be integrated provided the following simplification is made: if one uses a thin plate with thickness $L$ of the order
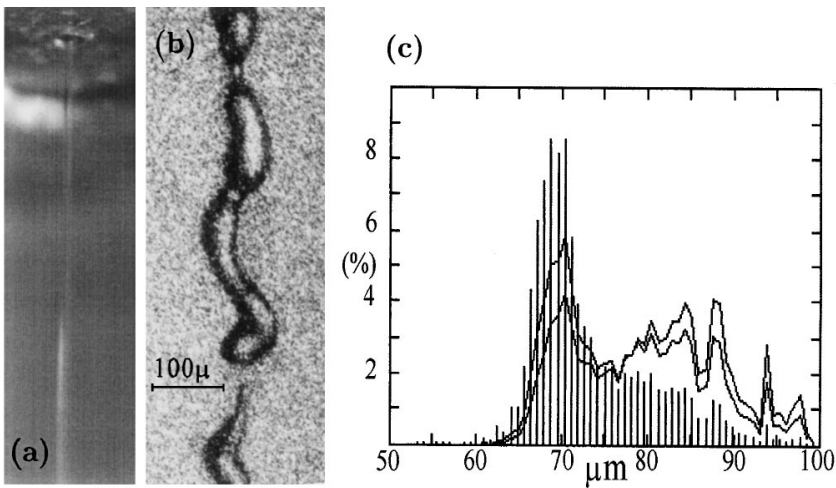

FIG. 3. (a) View of microjet near the exit of the orifice. Same liquid, geometrical parameters, and $d_{j}$ as in Fig. 2. $\Delta P_{g}=32.4 \mathrm{kPa}$ and $Q=8.2 \mu$ liter $/ \mathrm{s}$. (b) This photograph shows a less regular breakup pattern. (c) Droplet size histogram showing polydispersity. Standard deviation $\sigma=16.5 \%$. 
or smaller than the hole's diameter $D$ (which minimizes downstream perturbations in the gas flow), the pressure gradient up to the hole exit is on the average much larger than the viscous shear term $2 \tau_{s} / \xi$ owing to the surface stress. On the other hand, the axial viscous term [8-11] is of the order $O\left[\mu_{l} Q / D^{2} d_{j}^{2}\right]$, since the hole diameter $D$ is actually the characteristic distance associated with the gas flow at the hole's entrance in both the radial and axial directions. This term is very small compared to the pressure gradient in real situations, provided that $\Delta P_{g} \gg \mu^{2} / D^{2} \rho_{l}$ (which holds, e.g., for liquids with viscosities as large as 100 cpoises, using hole diameters and pressure drops as small as $D \sim 10 \mu \mathrm{m}$ and $\Delta P_{g} \simeq 100$ mbar). The neglect of all viscous terms in Eq. (4) is then justified. Notice that in this limit, the liquid flow is quasi-isentropic in the average (the liquid almost follows Bernoulli equation) as opposed to most micrometric extensional flows (e.g., [11,12]). Thus, integrating (4) from the stagnation region of both fluids up to the exit, one obtains a simple and universal expression for the jet diameter at the hole exit:

$$
d_{j} \simeq\left(\frac{8 \rho_{l}}{\pi^{2} \Delta P_{g}}\right)^{1 / 4} Q^{1 / 2},
$$

which for a given pressure drop $\Delta P_{g}$ is independent of geometrical parameters (hole and tube diameters, tube-hole distance, etc.), liquid and gas viscosities, and liquid-gas surface tension. This diameter remains almost constant up to the breakup point since the gas pressure after the exit remains constant.

Experimental analysis and comparison.-Fourteen different liquids whose properties are given in Table I have been used in our experimental study.

Three different plate hole diameters $D$, plate thicknesses $L$, tube radii $R_{o}(D=0.15,0.2$, and $0.3 \mathrm{~mm} ; L=$ $0.1,0.2$, and $0.35 \mathrm{~mm} ; R_{o}=0.2,0.4$, and $0.6 \mathrm{~mm}$, re-

TABLE I. Liquids used and some of their physical properties at $24.5^{\circ} \mathrm{C}\left(\rho: \mathrm{kg} / \mathrm{m}^{3}, \mu\right.$ : cpoise, $\left.\gamma: \mathrm{N} / \mathrm{m}\right)$. Also given, the symbols used in the plots.

\begin{tabular}{lrccc}
\hline \hline \multicolumn{1}{c}{ Liquid } & $\rho$ & $\mu$ & $\gamma$ & Symbol \\
\hline Heptane & 684 & 0.38 & 0.021 & $\bigcirc$ \\
Tap water & 1000 & 1.00 & 0.056 & $\diamond$ \\
Water + glycerol 90/10 v/v & 1026 & 1.39 & 0.069 & $\triangle$ \\
Water + glycerol 80/20 v/v & 1052 & 1.98 & 0.068 & $\nabla$ \\
Isopropyl alcohol & 755.5 & 2.18 & 0.021 & $\times$ \\
Water + glycerol 70/30 v/v & 1078 & 2.76 & 0.067 & + \\
Water + glycerol 60/40 v/v & 1104 & 4.37 & 0.067 & $\bullet$ \\
Water + glycerol 50/50 v/v & 1030 & 6.17 & 0.066 & $\bigcirc$ \\
1-Octanol & 827 & 7.47 & 0.024 & $\diamond$ \\
Water + glycerol 40/60 v/v & 1156 & 12.3 & 0.065 & $\triangle$ \\
Water + glycerol 35/65 v/v & 1167 & 15.9 & 0.064 & $\nabla$ \\
Water + glycerol 30/70 v/v & 1182 & 24.3 & 0.064 & $\times$ \\
Water + glycerol 25/75 v/v & 1195 & 38.7 & 0.063 & + \\
Propylene glycol & 1026 & 41.8 & 0.036 & $\bullet$ \\
\hline \hline
\end{tabular}

spectively), and several distances $H$ from the tube mouth to the orifice ranging from $H=0.5 \mathrm{~mm}$ to $H=1.5 \mathrm{~mm}$ have been used. The jet diameter has been measured at the hole exit [see Figs. 2(a) and 2(b)] and is plotted as a function of the pressure difference $\Delta P_{g}$ and flow rate $Q$ in Figs. 4(a) and 4(b), respectively. Although this technique allows for jet diameters even below one micron, larger flow rates and diameters have been used in this study to diminish the measuring errors.

In order to collapse all of the data, we define a reference flow rate $Q_{o}$ and diameter $d_{o}$ based on the minimal values, from expressions (3) and (5), that can be attained in stable regime for a given $\Delta P_{g}$ :

$$
Q_{o}=\left(\frac{\gamma^{4}}{\rho_{l} \Delta P_{g}^{3}}\right)^{1 / 2}, \quad d_{o}=\frac{\gamma}{\Delta P_{g}} .
$$

These definitions provide the advantage of a nondimensional expression for (5), as

$$
d_{j} / d_{o}=\left(8 / \pi^{2}\right)^{1 / 4}\left(Q / Q_{o}\right)^{1 / 2},
$$

which allows for a check for the validity of neglecting the surface tension term in (4) (i.e., $Q / Q_{o}$ should be large).

(a)

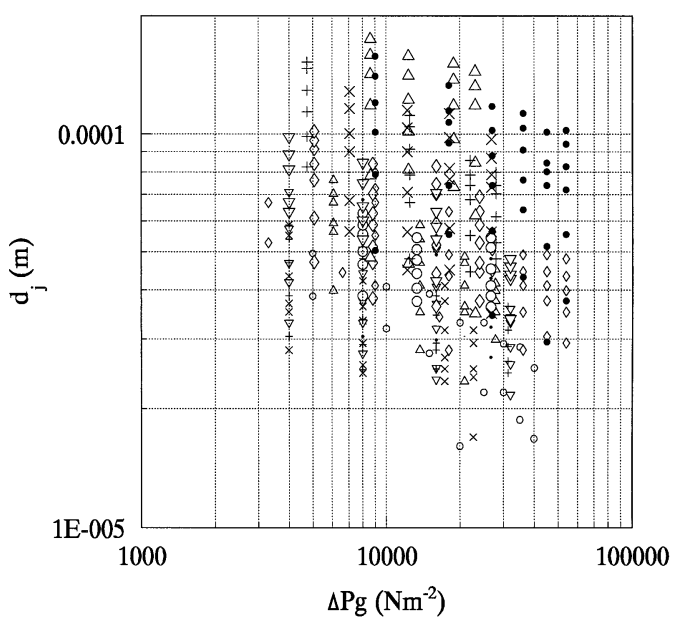

(b)

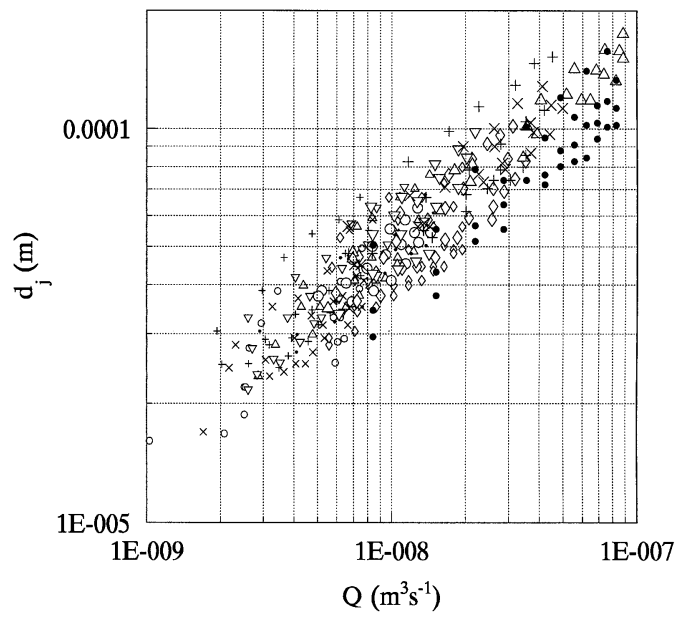

FIG. 4. (a) Measured jet diameter $d_{j}$ at the hole exit vs $\Delta P_{g}$, and (b) $d_{j}$ vs $Q$, for the fourteen liquids used and different geometrical configurations (see Table I). 


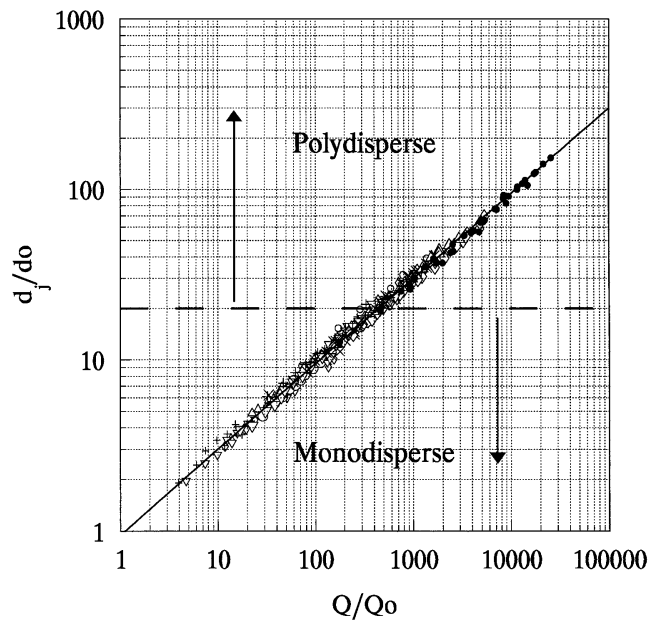

FIG. 5. Nondimensional plot of $d_{j} / d_{o}$ vs $Q / Q_{o}$. Continuous line shows theoretical prediction given by Eq. (7). Also shown are the parametric regions of monodispersity and polydispersity of the resulting spray.

Notice that if the measured $d_{j}$ follows expression (5), the surface tension cancels out in (7). Also notice that $d_{j} / d_{o} \simeq \mathrm{We} / 2$.

350 measured values of $d_{j} / d_{o}$ versus $Q / Q_{o}$ are plotted in Fig. 5. A continuous line represents the theoretical prediction (7), independent of liquid viscosity and surface tension. The use of different hole and tube diameters as well as tube-hole distances does not have any appreciable influence on $d_{j}$. The collapse of the experimental data and the agreement with the simple theoretical model is excellent. Finally, the experimental values of $Q$ are at least four times larger than $Q_{o}$ (being in most cases several hundreds times larger), which justifies the neglect of the surface tension term in Eq. (4).

The flow here reported shows robustness and versatility. The liquid jet does not touch the hole, neither does it experience the high shear associated with micrometric flows with a solid contact that may fracture or degrade some molecular species [3]. The plate holes are orders of magnitude larger, and the necessary pressures much smaller, than the ones necessary to achieve comparable liquid jets using direct liquid injection (e.g., [12]), which yields an important decreasing in clogging risks and other costs. The simple capillary tube-hole configuration can be packed and multiplexed with the only limit being set by manufacturing constraints.

As an additional application, we present in Fig. 6 a microscopic compound jet made of a low viscosity

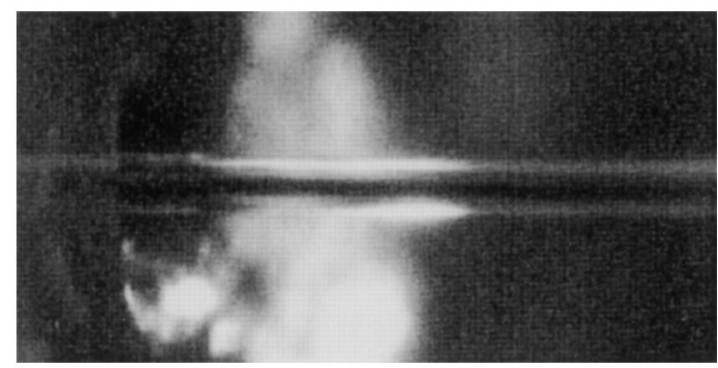

FIG. 6. Compound jet view at the hole's exit. Silicone oil jet surrounding a black ink jet. Arbitrary parameters.

silicone oil jet surrounding a water-based black ink thread. The injection occurred so that the black ink formed a steady cusplike meniscus inside the surrounding silicone meniscus. The resulting droplets were compound spheres consisting of a silicone cover encapsulating a black ink core. This method can be applied for manufacturing compound micropellets useful for targeted drug delivery, special material processing, etc.

The author is indebted to Professor Howard A. Stone for his insightful comments, interest, and for being the one who suggested the experiments which eventually shed light on the understanding of the phenomenon, and to Mr. Khalil Whitmore for his help in performing these experiments. This work is supported by the Spanish Comisión Interministerial de Ciencia y Tecnología, Project numbers PB93-1181 and PB96-1341.

[1] W.C. Hinds, Aerosol Technology (J. Wiley \& Sons, New York, 1982).

[2] A.M. Gañán-Calvo and A. Barrero, Spanish Patent No. P9601101 (1996); PCT Patent No. PCT/ES97/00034 (1997).

[3] K.D. Knudsen, M.C.L. Martínez, and J.G. de la Torre, Biopolymers 39, 435 (1996).

[4] R. F. Service, Science 277, 1199 (1997).

[5] T. Miyazaki and T. Kubo, J. Phys. Soc. Jpn. 64, 3275 (1995).

[6] J.R. Melcher and E.P. Warren, J. Fluid Mech. 47, 127 (1971).

[7] S. Bechtel, M. G. Forest, and K. J. Lin, Stability Appl. Anal. Continuous Media 2, 59 (1992).

[8] J. Eggers, Phys. Rev. Lett. 71, 3458 (1993).

[9] A. M. Gañán-Calvo, J. Fluid Mech. 335, 165 (1997).

[10] M.P. Brenner, J. Eggers, K. Joseph, S. R. Nagel, and X. D. Shi, Phys. Fluids 9, 1573 (1997).

[11] H. A. Stone, Annu. Rev. Fluid Mech. 26, 65 (1994).

[12] T. Hasegawa, M. Suganuma, and H. Watanabe, Phys. Fluids 9, 1 (1997). 